\title{
Improvement of Neutron Color Image Intensifier Detector using an Industrial Digital Camera
}

\author{
Takashi Kamiyama, a ${ }^{1,}$, Koichi Nittoh ${ }^{2, b}$ and Kazuyuki Takada ${ }^{3, c}$ \\ ${ }^{1}$ Faculty of Engineering, Hokkaido University, Kita 13 Nishi 8, Kita-ku, Sapporo 060-8628, \\ Hokkaido, Japan \\ ${ }^{2}$ Toshiba Technical Services International Corp., 8 Shinsugita, Isogo-ku, Yokohama 235-8523, \\ Kanagawa, Japan \\ ${ }^{3}$ TAKADA KIKAI Co., Ltd., 5, Kita 3 Higashi 4, Chuo-ku, Sapporo 060-0033, Hokkaido Japan \\ atakashik@eng.hokudai.ac.jp, ${ }^{b} k$ koichi1.nittoh@glb.toshiba.co.jp, ${ }^{c}$ takata-k@sea.plala.or.jp
}

Keywords: Neutron Radiography, Image Intensifier, CMOS Camera, Peltier Cooling

\begin{abstract}
Among various kinds of detectors being developed for neutron transmission imaging, Neutron Image Intensifier (NII) is still a promising option in terms of field of view and sensitivity. Since NII has a position resolution higher than $0.1 \mathrm{~mm}$, it can be expected to obtain a high-sensitivity and high-gradation fine image with a camera to be combined [1]. In this research, we selected CMOS cameras for industrial applications with linearity over a wide sensitivity range, and examined the performance of the camera, especially the cooling effect of the sensor which is important in long-time measurement. Based on this result, we developed a portable imaging system that compactly integrates a cooled camera with NII, and constructed a system that can be used quickly in various neutron facilities.
\end{abstract}

\section{Introduction}

In recent years, with the progress in compact neutron facilities development, an application of high-sensitive Neutron Image Intensifier (NII) is expected for neutron imaging under low flux. NII is a vacuum tube that converts an input neutron image to a visible image [1, 2]. Neutrons react at the NII incident window and produce charged particles, which are converted into fluorescence and electrons at the subsequent phosphor and photoelectric layers, respectively. The emitted electrons are accelerated by the electric field toward the phosphor luminescent window while maintaining the neutron incident image. For the neutron detection by the image intensifier some developments were necessary in the input and output windows with short decay time to apply to pulsed neutron sources [3, 4].

As the imaging system the NII is combined with an optical system which consists of a lens and a camera. NII can adapt a kind of different cameras such as single lens reflexes, high speed cameras, video cameras, etc., but the current whole detector system size with neutron shielding containers becomes large, then it is not easy to carry and setup it for experiments. Also, in case of the NII imaging with a digital camera, we should consider the noise due to increasing the camera amplifier gain besides the radiation origin. Such noise is unique to high-sensitivity digital cameras, and we call it as high-sensitivity noise in this paper.

Against to these issues, we improved the NII imaging system. Here, we introduce the developed system which is portable so that on-site imaging is possible and which enables stable imaging with less noise even for long-time exposure.

\section{Selection of digital camera}

In proper condition of the camera combined with our NII is to have a $1: 1$ aspect ratio to maximize the effective field of view, because the origin of the NII is in the Color Image 
Intensifier (CII) for X-ray [5], which has a circular output fluorescent screen. As the NII camera is remotely operated and displayed, USB-3.0 compatible camera which has a transfer rate above $60 \mathrm{fps}$ and more than 2,076,600 pixels equivalent to the current HDTV is preferable, because it can handle video and operation with a single cable at high communication speed. By integrating short exposure time images with a high-speed compact monitoring video camera, it is possible to suppress noise and take a wide luminance range than using a single lens reflex camera with long exposure time. We also created capturing software at 32 bits/pixel to expand the dynamic range of integrated images. We conducted tests and built-in designs using cameras of following two types,

Type-A: CMOSIS CMV-4000, USB-3.0, square 4.19 million pixels view field, up to 90 fps,

Type-B: SONY IMX250, USB-3.0, square 5.01 million pixels view field, up to 75 fps.

We consider three kinds of noise. In case of long-time exposure in digital camera photography, the imaging sensor (CCD or CMOS) generates unnecessary electrons, so called dark current noise, which leads to bright spots on the screen. Also, amplifier noise and heat fog occur from the heat generation of the electronic circuit, and appears on the monitor as pink as the background on the entire screen. High sensitivity noise is caused by raising the ISO sensitivity or gain of the digital camera against dark images. Generally, in digital cameras, the last noise is dominant when imaging a dark subject in a short time, which leads to gritty images. When combined with long-time exposure, dark current noise becomes conspicuous especially. Many cameras are equipped with a noise reduction function that cancels above mentioned noises. Especially, the dark current noise is unique to the camera, but the noise has regularity. Namely, when imaging under the same condition, the noise occurs in the same position. Therefore, it is possible to reduce the dark current noise by imaging with the shutter closed under the same condition and subtracting the noise image (dark current correction), though the imaging time becomes doubled. In the case of repeated imaging with the same setting, efficiency can be improved by reuse of dark current correction data. However, the processing is not appropriate when the imaging conditions changed due to the long time operation. That is, the noise pattern varies by amplifier noise or heat fog and the subtraction is not always valid, so that it is important to keep the temperature of the camera constant.

The above-mentioned noise related issues are similarly examined for astronomical photography camera. In recent digital cameras, high sensitivity noise has been decreasing due to the evolution of the type of image pickup sensor, the circuit configuration and the image processing engine. However, it is necessary to note that the dynamic range is narrowed by these image processing operations.

The camera noise related common issue is the temperature of the camera. Cooled CCD cameras are used as a long-time imaging camera for astronomical photography. They have not yet been introduced for NII because of its high price and rapid advancement of camera sensors and difficulty in dealing with new models. In this system, we evaluated the characteristics of general surveillance cameras with added cooling function to them for improving the performance of stable operation, and the change in characteristics before and after cooling.

The relationship between neutron flux and imaging time is as follows. For example, if neutron transmission images can be continuously taken with a moving average of 1 second at 30 fps under the flux of $3 \times 10^{6} \mathrm{n} / \mathrm{cm}^{2} / \mathrm{sec}$ as typical small reactors, it would take as much as 1000 seconds (17 minutes) under flux $3 \times 10^{3} \mathrm{n} / \mathrm{cm}^{2} / \mathrm{sec}$ to get the similar brightness single frame image, and 10000 seconds (2.8 hours) under flux $3 \times 10^{2} \mathrm{n} / \mathrm{cm}^{2} / \mathrm{sec}$ as neutron generators, respectively. Cameras installed in the previous NII systems were video cameras, high-definition digital single-lens reflex cameras or high-speed cameras [3, 4]. In case of exposing with 17 minutes or 2.8 hours in the single lens reflex camera, bulb shooting will be performed during that 
time. In high-speed cameras, assuming that the maximum exposure time is 15 seconds, it is necessary to accumulate 67 images in 17 minutes or accumulate 667 images in 2.8 hours. Again, keeping the temperature of the camera stable during these measurements is essential for noise processing.

\section{Relationship between camera temperature and dark current image}

Transition characteristics of the camera temperature and image brightness after turning on the camera power were measured for the Type-A camera. Each camera image was accumulated during the elapsed time with external light blocked by the lens cap. Under the fixed condition of 15 seconds of camera exposure time, the initial image under ambient temperature of about $14^{\circ} \mathrm{C}$ was just black, but after longer elapsed time it became gray as the result of the camera temperature increasing. The camera temperature rose sharply to near $35^{\circ} \mathrm{C}$ in about 30 minutes after turning on the camera power. We repeated background imaging in every 30 minutes with accumulating 1800 images at a high camera gain and 1 second exposure time. The result is as shown in Fig. 1, the background RGB component of average brightness for elapsed time is increasing due to the increase of the dark current noise.

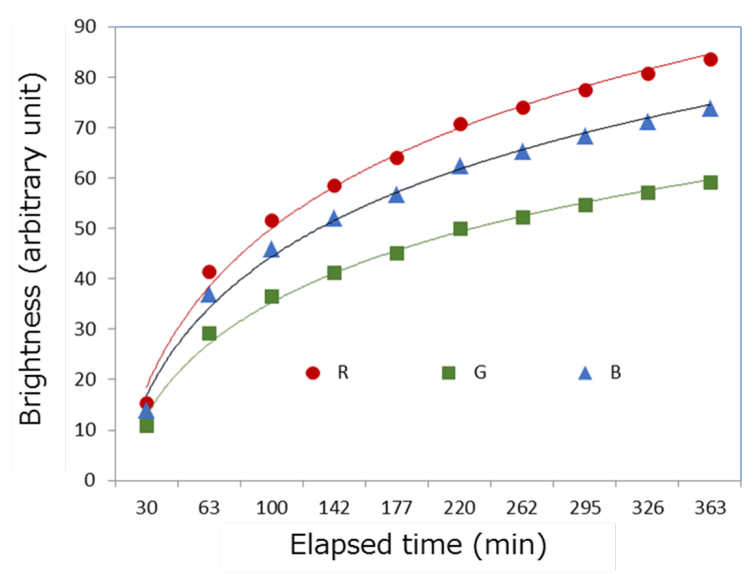

Fig.1. Elapsed time dependence of dark current image of Type-A camera [8].

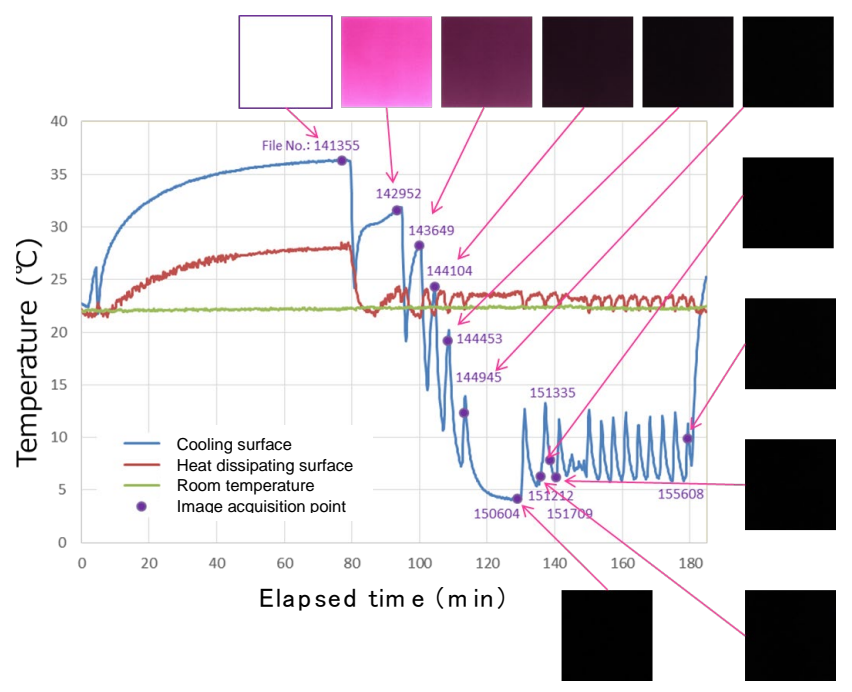

Fig.3. Dark current images and temperature dependence of Type-A camera [8]. (a) Water-cooled type

(b) Air-cooled type

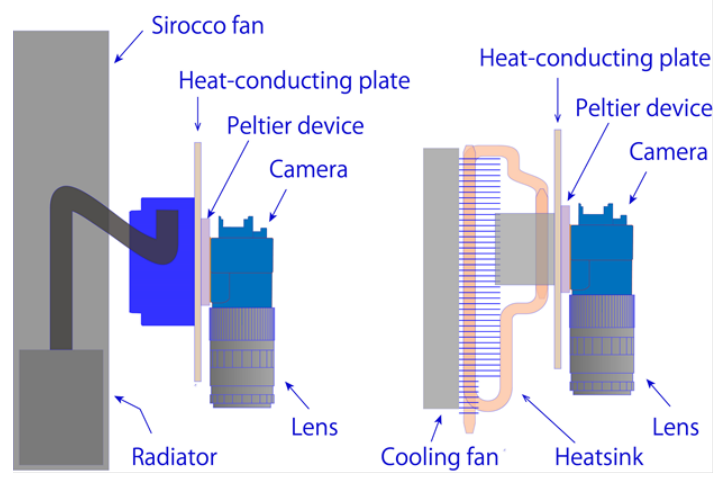

Fig.2. Camera cooling systems.

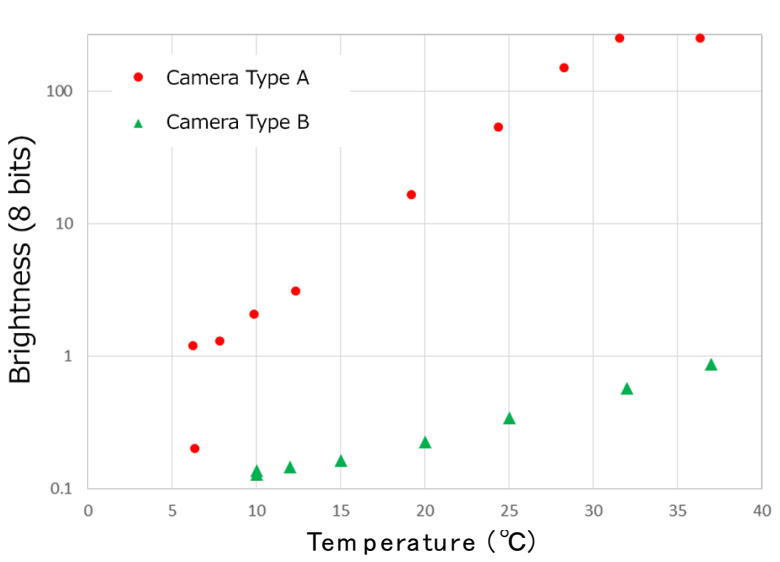

Fig.4. Temperature characteristics of the Type-specific cameras [8]. 
In order to maintain the camera body at a low temperature, a Peltier device was applied. The heat-conducting plate which receives heat from the Peltier device is cooled either by using a water-cooling type (Fig. 2(a)) or an air-cooling type (Fig. 2(b)). Using these simple cooling systems, the cooling performance was measured without turn-on the camera. Both Peltier devices used were 6A type and the measurement was carried out under fixed conditions of an applied voltage of $5 \mathrm{~V}$ and a steady state current of $1.5 \mathrm{~A}$. Measurements were carried out in the ambient temperature of about $24^{\circ} \mathrm{C}$ in both cases. However, the air-cooling type was confirmed that the temperature on both sides on the heat-conducting plate of the Peltier device and the heat radiator changed largely against slight change in room temperature. Namely, among these two types of coolers, it was shown that the water-cooling type is stable and the cooling capacity is higher. The relationship between camera temperature and dark current image was measured with the camera power on and the Peltier device operated at 5V (1.5A) together with the water-cooling unit active. The measured results are shown in Fig. 3. The operation was started with the cooling unit stopped. When the camera temperature exceeded $35^{\circ} \mathrm{C}$, the Peltier cooling unit was repeated manually turn on and off, and the dark current images of 15 second exposure time were acquired at the points marked closed circle dropped. The noise decreased from the white image with more than 250 in 8 bit brightness range at $37^{\circ} \mathrm{C}$ to gray then black image with decreasing temperature.

About the new Type-B camera, images of the dark current noise against the camera temperature were measured similarly with the Type-A. Although the noise of the Type-B increases as the camera temperature rises, the noise component is drastically smaller than the Type-A. Figure 4 shows the temperature dependence of the image brightness taken by the Type$\mathrm{B}$ together with that by the Type-A. Under Peltier conditions cooled to $15^{\circ} \mathrm{C}$ or less, it was confirmed that the dark current noise of the Type-A was $1 \%$ or less than that at room temperature. On the other hand, by the Type-B it was suppressed to $0.1 \%$ or less even at $20^{\circ} \mathrm{C}$. Then we adopted the Type-B camera.

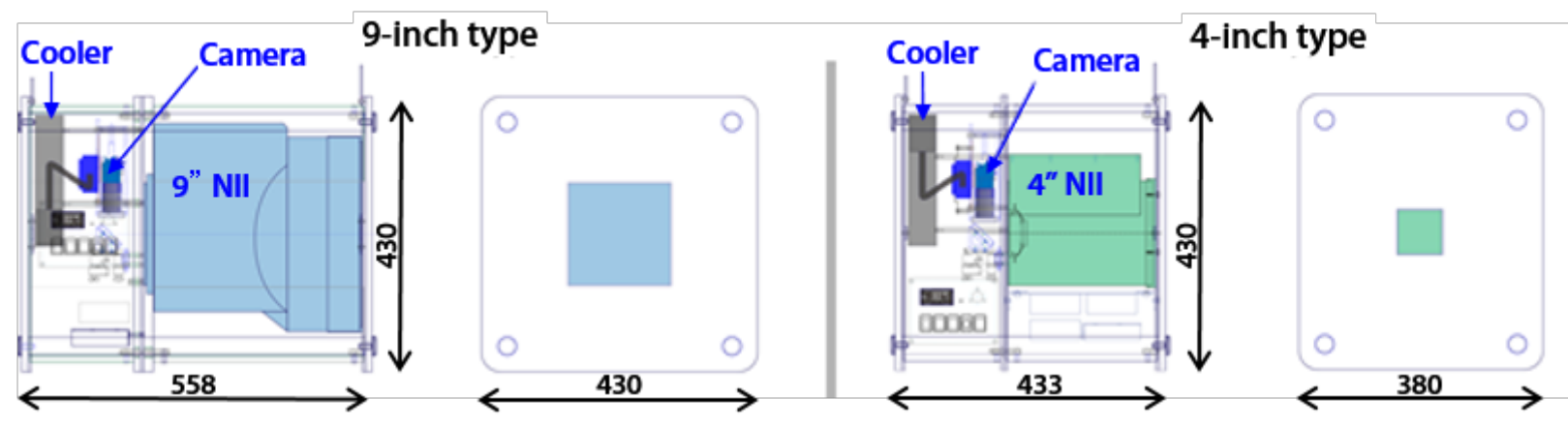

Fig.5. Design drawings of 9 and 4-inch NII systems.

\section{Incorporation of cooled camera into NII}

An imaging system combined the NII with the cooled camera has to be newly designed and fabricated in consideration of radiation shielding. In order to make the system as compact as possible, it is desirable to reduce the distance from the NII output phosphor screen to the camera. In the actual optical design, equipment placements were decided considering the size of a lens, a mirror, the camera and the cooling unit, as well as their radiation shielding arrangement. In particular, the camera was placed right angle bent by the mirror to prevent direct hits from the incident neutron irradiation. Figure 5 shows the camera built-in configuration of 4-inch and 9inch NII system, respectively. The container of the system is covered with neutron shielding material of $50 \%$ boron carbide in epoxy resin. 


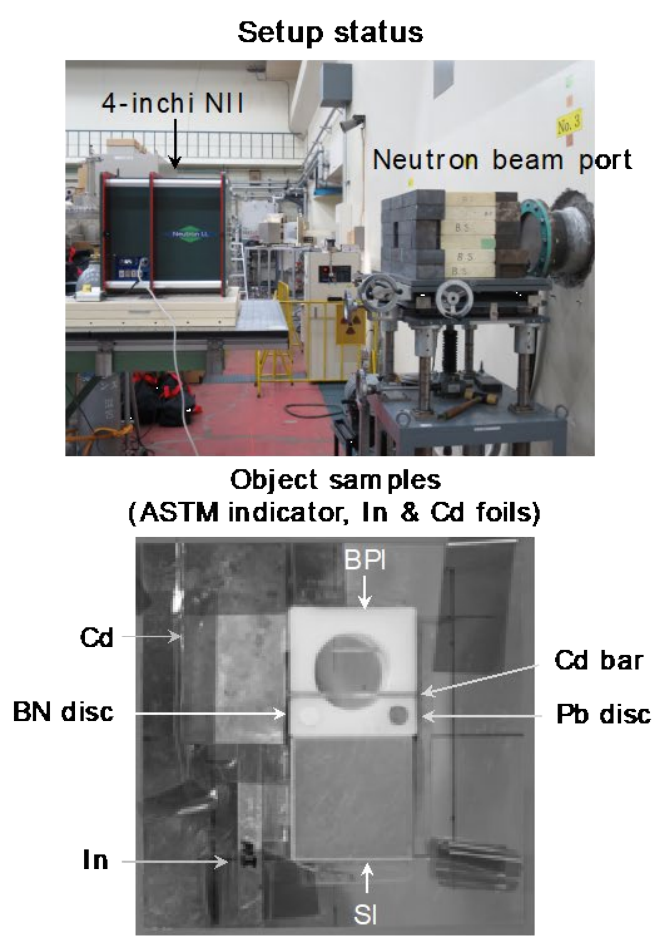

(a) Gamma-burst image

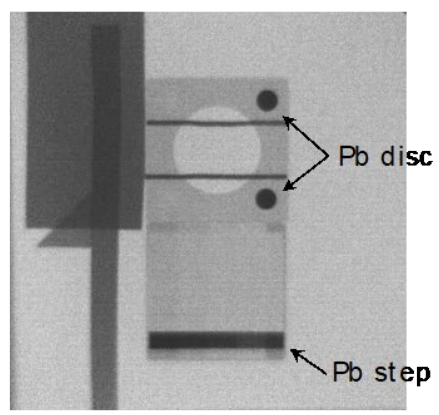

(c) $\mathrm{Pb} \& \mathrm{~B}_{4} \mathrm{C}$ filter im age

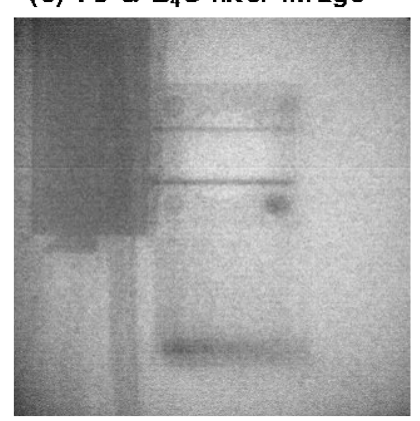

(b) Pb-10m filter image

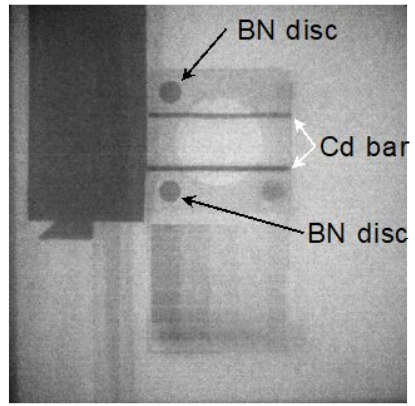

(d) Difference neutron image

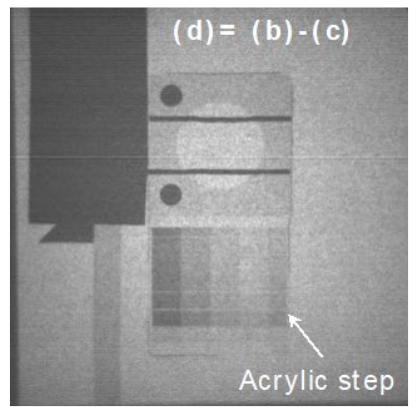

Fig.6. Neutron imaging results at HUNS.

Figure 6 shows the results of imaging using the Hokkaido University neutron source (HUNS), which is a compact accelerator pulsed source. In the measurement, we used BPI and SI indicators according to ASTM standard as shown in the lower left of Fig. 6. Because HUNS is the pulsed neutron source using an electron linac, intense burst X-rays are initially produced besides neutrons. In the case of missing lead filter as a shield for X-rays in front of the NII input window, an image by the burst X-rays is dominant as shown in Fig. 6 (a) where the upper two lead discs and the lower horizontal lead step can recognize. Inserting a $10 \mathrm{~mm}$ lead filter results as Fig. 6 (b). The image of the lead discs becomes paler, and two discs of boron nitride turn visible on the left side. Further, an image in which a boron filter was inserted is shown in Fig. 6(c), and from here it can be confirmed the weakened irradiations of both X-ray and neutron. By subtracting Fig. 6 (c) from Fig. 6 (b), the image of Fig. 6 (d) is obtained of a neutron image. In this last image, there were no shadows of the lead parts, but an image of the boron nitride discs, the step of cadmium and polyethylene were confirmed.

Figure 7 shows the variation of brightness by changing the camera temperature from 10 to $39^{\circ} \mathrm{C}$ with the same setup of Fig. 6. We set an ROI on the lead step wedge of the SI indicator, then the step-like brightness changes were determined depending on the temperature. The right side of Fig. 7 shows the ROI brightness normalized to 1 at an outside place of the samples. When the camera temperature is $15^{\circ} \mathrm{C}$ or less, the brightness deviation is less than $1 \%$, but with increasing its temperature the deviation rises such as $1.3 \%$ at $25^{\circ} \mathrm{C}$ and $3.8 \%$ at $39^{\circ} \mathrm{C}$. This result includes the fluctuation of the neutron source itself as well as the fluctuation due to the temperature control of the camera. 

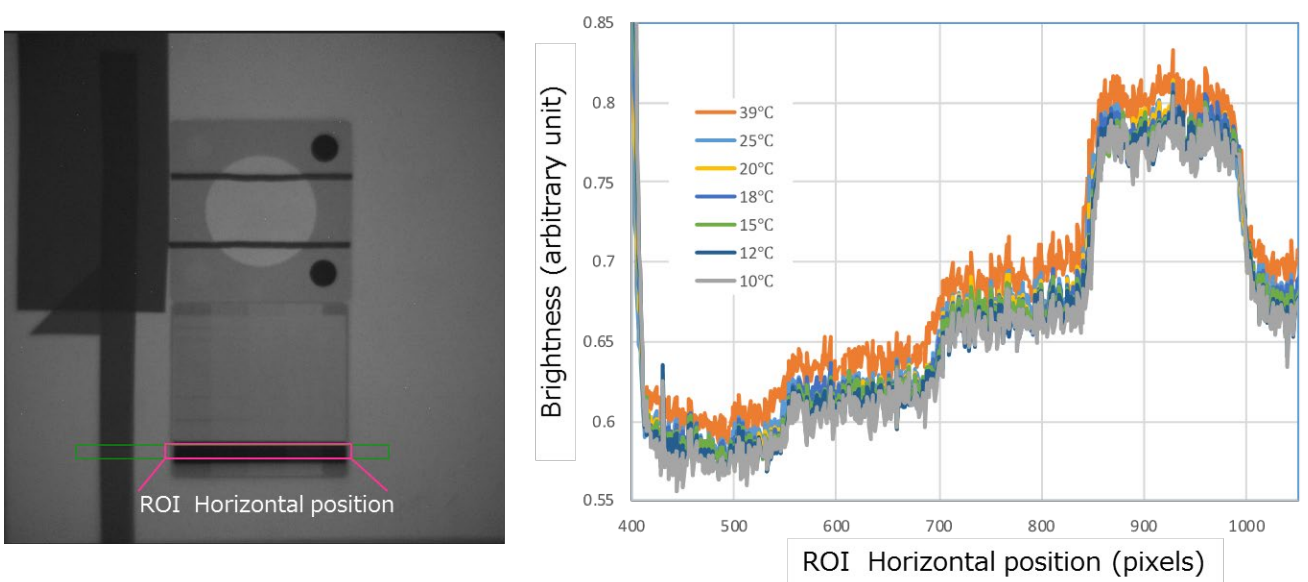

Fig.7. Change the status of the brightness values of the image with a change in temperature of the camera.

Figure 8 shows the results of the brightness change for 50 minute accumulation in 16 bits using the 9-inch NII under $12^{\circ} \mathrm{C}$ control. It is confirmed that the amounts of the fluctuation are \pm 195 with respect to the average brightness of 30302.5, so it means the stable measurement is performed within the $1.3 \%$ fluctuation, which included the neutron source instability.

\section{Conclusion}

We have developed a compact NII system aiming to stable operation for a long-time while reducing noise. In particular, with a

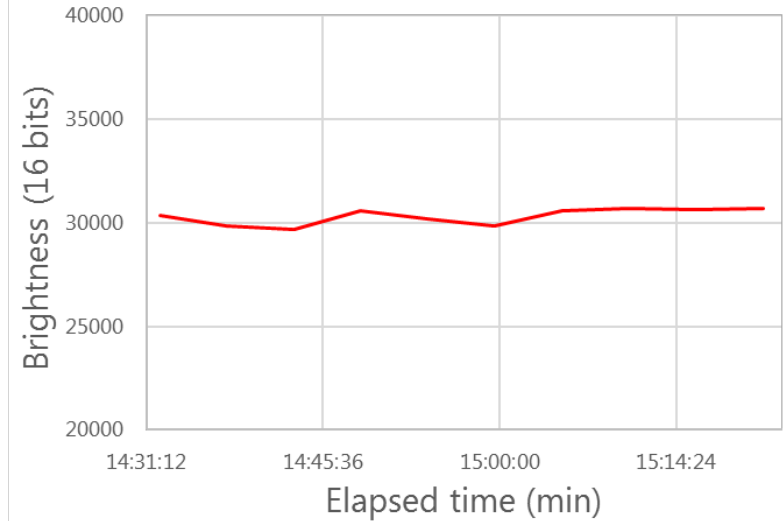

Fig.8. Stability check of 9-inch NII camera temperature setting. low neutron flux or a subtle change imaging, it is necessary to perform long-time exposure imaging and multiple image accumulation since the variation in the number of neutrons is small. For the purpose, we selected industrial CMOS cameras with linearity over a wide sensitivity range, and examined the performance of the cameras, especially the cooling effect which is important in the long-time measurement. Through the experiments, it became clear that there was a difference in temperature dependence on the types of imaging CMOS device in the camera, and it was also understood that the temperature characteristics differ depending on the cooling method. Based on the above results, we have developed a portable system with the compact neutron shielding. As a result, we succeeded in developing a prototype NII system that operates stably and is not susceptible to temperature changes.

\section{Acknowledgements}

This research is partially supported by "AS272I001c, Adaptable and Seamless Technology Transfer Program through Target-driven R\&D, JST", by “the Development of Non-Destructive Methods Adapted for Integrity test of Next generation nuclear fuels project by the Ministry of Education, Culture, Sports, and Technology (MEXT), Japan”, and by “JSPS KAKENHI Grant Number 17H03515”. 


\section{References}

[1] K. Nittoh, Neutron Color Image Intensifier, Hamon, 22 (2012) 322-328 [in Japanese]. https://doi.org/10.5611/hamon.22.4_322

[2] K. Nittoh, C. Konagai, T. Noji, K. Miyabe, New feature of the neutron color image intensifier, Nucl. Instr. and Meth. A, 605 (2009) 107-110.

https://doi.org/10.1016/j.nima.2009.01.136

[3] K. Nittoh, C. Konagai, M. Yahagi, Y. Kiyanagi, T. Kamiyama, Development of Neutron Color Image Intensifier for Pulsed Neutron Source, Physics Procedia, 69 (2015) 177-184. https://doi.org/10.1016/j.phpro.2015.07.025

[4] K. Mochiki, K. Ishizuka, K. Morikawa, T. Kamiyama, Y. Kiyanagi, Development of a New High-Frame-Rate Camera for Pulsed Neutron Transmission Spectroscopic Radiography, Physics Procedia, 69 (2015) 143-151. https://doi.org/10.1016/j.phpro.2015.07.021

[5] K. Nittoh, C. Konagai, T. Noji, Development of multi-color scintillator based X-ray image intensifier, Nucl. Instr. And Meth. A, 535 (2009) 686-691. https://doi.org/10.1016/S01689002(04)01658-4

[6] ASTM Designation, Standard Method for Determining Image Quality in Direct Neutron Radiographic Examination, E545-91 (1991)

[7] Riso National Laboratory, Standardization Activities of the Euratom Neutron Radiography Working Group, RISO-M-2356 (1982)

[8] T. Kamiyama, K. Nittoh, Consideration of the neutron image intensifier camera, Hamon, 28 (2018) 77-83 [in Japanese] 